\title{
Production of Higher Alcohols and Short Chain Fatty Acids by Different Yeasts Used in Rum Fermentations
}

\author{
L. FAHRASMANE, A. PARFAIT, C. JOURET, and P. GALZY
}

\begin{abstract}
The production of higher alcohols and short chain fatty acids by yeasts used in rum fermentations was greater by the Saccharomyces strains than by those belonging to the Schizosaccharomyces genus. There were also qualitative differences between these two genera. Among the organic acids of the raw material, citric acid and cisaconitic acid constitute a special feature of the composition of the resulting rums. Their presence entails the abundant production of biomass. Citric acid alone induced acrylic acid formation by strains of two species among the three studied.
\end{abstract}

\section{INTRODUCTION}

YEAST SEEDING with baker's yeast is becoming a general practice in the distilleries of Guadeloupe and Martinique. At the turn of this century, the strains of Schizosaccharomyces were dominant in the "spontaneous" fermentation of media for rum manufacture (Pairault, 1903; Allan, 1906), these were taken over by Saccharomyces strains due to modifications of different fermentation parameters. However, the former strains can still be found in the unseeded media of small distilleries in Haiti. This evolution of the yeast flora has modified the flavor of rum due to genus characteristics and to a lesser extent, to species and strain characteristics in the production of secondary products. The criteria in use at the present time for the selection of sugar cane cultivars take into account the requirements of the sugar refinery industry:sugar content of the raw material. Compounds other than sugar which contribute to the flavor of rum are little studied. These compounds may explain some particular features of rum compared to other alcoholic beverages (Suomalainen, 1975). For instance, a study of Parfait et al. (1972) showed the favorable effect of cane wax on the ester fraction of rum.

In the present work, we report a study of the production of short chain fatty acids and higher alcohols by pure yeast cultures, and the influence of medium composition on the formation of these flavor components.

\section{MATERIALS \& METHODS}

WE USED YEAST STRAINS that were isolated from unseeded media in rum manufacture, maintained in the collection of the Laboratoire de Technologie INRA Guadeloupe. These included: Saccharomyces cerevisiae Hansen 493 and 390; Saccharomyces chevalieri Guillermond 467 and 377; Schizosaccharomyces pombe Lindner (these strains were isolated in Guadeloupe and in Martinique); Schizosaccharomyces pombe Lindner 850, isolated in Haiti; and Saccharomyces cerevisiae Hansen Lb, a commercial active dry yeast.

The precultures were grown in strongly aerated malt-Wickerham medium $(20 \mathrm{~g} / \mathrm{L}$ saccharose $)$.

Authors Fahrasmane and Parfait are affiliated with the Centre de Recherches Agronomiques des Antilles et de la Guyane, 97170 petit-Bourg Guadeloupe F.W.I. Author Jouret is affiliated with the Institut National de la Recherche Agronomique. Station de Technologie, 31320 Auzeville, France. Author Galzy is affiliated with Laboratoire de la Chaire de Génétique et Microbiologie, Ecole Nationale Supérieure Agronomique, 34060 Montpellier, Cedex France.
The fermentation tests were performed in $500 \mathrm{~mL}$ flasks containing $250 \mathrm{~mL}$ medium, under anaerobic conditions but not under nitrogen atmosphere. The standard conditions were as follows: $\mathrm{pH} 5.5$; temperature $30 \pm 1^{\circ} \mathrm{C}$, seeding yeast dry matter $1 \mathrm{~g} / \mathrm{L}$; saccharose $100 \mathrm{~g} / \mathrm{L}$.

The media used were: (1) A synthetic medium: the requirement for a well defined and reproducible medium during the whole experiment led us to use the Oura synthetic medium (1974) containing $\left(\mathrm{NH}_{4}\right)_{2} \mathrm{SO}_{4}(1.2 \mathrm{~g} / \mathrm{L})$ and saccharose $(100 \mathrm{~g} / \mathrm{L})$. Ergosterol and Tween 80 were added according to the concentrations given by Bauchop and Elsden (1960). (2) Sugar cane juice was obtained by pressing fresh canes; the juice was preserved in a freezer at $-15^{\circ} \mathrm{C}$. The total acidity was $19.5 \mathrm{meq} / \mathrm{L} ; \mathrm{pH} 5.8$. The cane juice composition is shown in Table 1. For fermentation purposes this cane juice was dilu ted to obtain $100 \mathrm{~g} / \mathrm{L}$ sugars. (3) Supplemented cane juice: the above medium was supplemented with $\left(\mathrm{NH}_{4}\right)_{2} \mathrm{SO}_{4}$ to give $250 \mathrm{mg} / \mathrm{L}$ nitrogen and with $\mathrm{KH}_{2} \mathrm{PO}_{4}$ to give $120 \mathrm{mg} / \mathrm{L}$ phosphorus at the beginning of the fermentation. The addition of nitrogen and phosphorus to the medium is a common practice in rum manufacturc.

Biomass was determined by centrifuging out the cells. The pellet was redispersed with distilled water and dried to constant weight at $108^{\circ} \mathrm{C}$.

Gas chromatographic methods were used for the assay of short chain fatty acids (Fahrasmane et al., 1983) and the assay of higher alcohols (Repression des Fraudes, 1973).

\section{RESULTS}

\section{Biomass production}

At the end of the fermentation, the resulting biomass was much less in the Oura synthetic medium than in the cane juice medium (Table 2). The greatest biomass occured in the supplemented cane juice. Biomass measurements for the cane juice medium were similar to the figures actually

Table 1-Composition of the cane juice used

\begin{tabular}{lclc}
\hline \multicolumn{4}{c}{ COMPONENTS } \\
\hline \multicolumn{1}{c}{$\mathrm{g} / \mathrm{L}$} & \multicolumn{3}{c}{ Amino acids $(\mathrm{mg} / 100 \mathrm{~mL}$ ) } \\
\hline Saccharose & 206.0 & Aspartic acid & 18.4 \\
Fructose & 0.7 & Threonine & 2.8 \\
Glicose & 3.5 & Serine & 7.2 \\
Acunitic acid & 0.12 & Asparagine & 82.6 \\
Citric acid & 0.12 & Glutanic acid & 0.5 \\
Ash & 2.5 & Glutamine & 22.1 \\
Nitrogen & 0.15 & Glycine & 0.9 \\
Phosphorus & 0.07 & Alanine & 12.2 \\
Potassium & 1.31 & Cystine & Traces \\
Calcium & 0.13 & Valine & 4.4 \\
Magnesilim & 0.27 & Methionine & 1.0 \\
Manganese & 0.2 & Leeicine & 0.91 \\
Sodium & 0.2 & Tyrosine & 1.2 \\
& & Amino-butyric acid & 6.9 \\
& & Histidine & 2.1 \\
& & Ornithine & Traces \\
& & Lysine & 1.4 \\
& & Ethanolamine & 0.1 \\
& & Arginine & 0.7 \\
& & Isolelicine & 1.5 \\
& & Phenyialanine & 0.7 \\
& & Total & 167.61 \\
\hline
\end{tabular}


AROMATIC COMPOUNDS FROM YEASTS IN RUM ...

Table 2-Effect of the organic acids on the production of biomass, the fermentation period and the production of higher alcohols ${ }^{\mathrm{a}}$

\begin{tabular}{|c|c|c|c|c|c|c|c|c|}
\hline \multirow[b]{2}{*}{ Strains } & \multirow[b]{2}{*}{ Mediab $^{b}$} & \multirow{2}{*}{$\begin{array}{l}\text { Yeast dry } \\
\text { matter }(\mathrm{g} / \mathrm{L})\end{array}$} & \multirow{2}{*}{$\begin{array}{l}\text { Period } \\
\quad(h r)\end{array}$} & \multicolumn{5}{|c|}{ Higher alcohois (mg/l) } \\
\hline & & & & Propanol & Isobutanol & Butanol & Isopentanol & Total \\
\hline S. cerevisiae Lb & $\begin{array}{l}\text { J } \\
\text { C } \\
\text { o }\end{array}$ & $\begin{array}{l}5.2(0.5)^{c} \\
7.5(3.5) \\
2.3(1.0)\end{array}$ & $\begin{array}{l}70(0.0) \\
65(3.0) \\
96(2.0)\end{array}$ & $\begin{array}{l}21(6) \\
40(9) \\
48(6)\end{array}$ & $\begin{array}{l}14(9) \\
23(8) \\
13(12)\end{array}$ & $\begin{array}{l}3(6) \\
2(12) \\
2(0)\end{array}$ & $\begin{array}{l}48(12) \\
35(7) \\
35(6)\end{array}$ & $\begin{array}{r}86(8) \\
100(8) \\
98(7)\end{array}$ \\
\hline S. cerevisiae 493 & $\begin{array}{l}J \\
c \\
0\end{array}$ & $\begin{array}{l}6.0(0.5) \\
8.9(1.0) \\
2.8(1.0)\end{array}$ & $\begin{array}{r}75(1.0) \\
65(1.0) \\
110(3.3)\end{array}$ & $\begin{array}{l}25(9) \\
40(4) \\
30(10)\end{array}$ & $\begin{array}{l}18(6) \\
34(6) \\
16(9)\end{array}$ & $\begin{array}{l}1(8) \\
2(4) \\
3(9)\end{array}$ & $\begin{array}{l}55(9) \\
55(9) \\
35(10)\end{array}$ & $\begin{array}{c}99(9) \\
131(6) \\
85(10)\end{array}$ \\
\hline S. cerevisiae 390 & $\begin{array}{l}\mathrm{J} \\
\mathrm{C} \\
\mathrm{O}\end{array}$ & $\begin{array}{l}4.6(1.5) \\
6.2(1.5) \\
3.0(1.0)\end{array}$ & $\begin{array}{l}70(0.0) \\
67(1.0) \\
76(1.5)\end{array}$ & $\begin{array}{l}21(11) \\
43(8) \\
34(7)\end{array}$ & $\begin{array}{l}12(13) \\
45(5) \\
19(10)\end{array}$ & $\begin{array}{l}6(7) \\
4(5) \\
2(12)\end{array}$ & $\begin{array}{l}49(5) \\
74(2) \\
47(12)\end{array}$ & $\begin{array}{c}98(8) \\
166(6) \\
107(10)\end{array}$ \\
\hline S. chevalieri 377 & $\begin{array}{l}J \\
C \\
0\end{array}$ & $\begin{array}{l}6.2(1.0) \\
8.6(0.0) \\
3.3(1.0)\end{array}$ & $\begin{array}{l}70(1.0) \\
64(1.5) \\
94(2.5)\end{array}$ & $\begin{array}{l}22(7) \\
35(8) \\
44(12)\end{array}$ & $\begin{array}{l}13(8) \\
24(6) \\
23(5)\end{array}$ & $\begin{array}{l}2(6) \\
2(6) \\
2(0)\end{array}$ & $\begin{array}{l}58(6) \\
56(9) \\
41(14)\end{array}$ & $\begin{array}{c}95(6) \\
117(8) \\
110(12)\end{array}$ \\
\hline S. chevalieri 467 & $\begin{array}{l}J \\
C \\
0\end{array}$ & $\begin{array}{l}6.9(0.5) \\
8.1(1.0) \\
3.1(1.0)\end{array}$ & $\begin{array}{l}70(1.5) \\
64(3.5) \\
95(1.0)\end{array}$ & $\begin{array}{l}36(11) \\
65(11) \\
40(5)\end{array}$ & $\begin{array}{l}30(9) \\
48(10) \\
33(5)\end{array}$ & $\begin{array}{l}1(12) \\
2(8) \\
3(12)\end{array}$ & $\begin{array}{l}68(12) \\
66(9) \\
36(1)\end{array}$ & $\begin{array}{l}135(11) \\
181(10) \\
111(6)\end{array}$ \\
\hline Sch. pombe G & $\begin{array}{l}J \\
\mathrm{C} \\
\mathrm{O}\end{array}$ & $\begin{array}{l}3.2(1.0) \\
4.4(1.0) \\
2.5(1.5)\end{array}$ & $\begin{array}{r}105(5.0) \\
90(3.5) \\
170(1.0)\end{array}$ & $\begin{array}{r}8(6) \\
10(9) \\
9(4)\end{array}$ & $\begin{array}{l}0 \\
0 \\
0\end{array}$ & $\begin{array}{l}1(7) \\
3(12) \\
1(12)\end{array}$ & $\begin{array}{l}1(12) \\
2(14) \\
3(9)\end{array}$ & $\begin{array}{l}10(7) \\
15(10) \\
13(5)\end{array}$ \\
\hline Sch. pombe 850 & $\begin{array}{l}J \\
C \\
O\end{array}$ & $\begin{array}{l}2.9(0.0) \\
3.8(1.0) \\
2.6(2.5)\end{array}$ & $\begin{array}{l}125(3.0) \\
120(4.0) \\
160(3.0)\end{array}$ & $\begin{array}{r}8(6) \\
12(9) \\
10(9)\end{array}$ & $\begin{array}{l}0 \\
0 \\
0\end{array}$ & $\begin{array}{l}1(4) \\
1(6) \\
3(6)\end{array}$ & $\begin{array}{l}3(11) \\
3(8) \\
6(6)\end{array}$ & $\begin{array}{l}12(7) \\
16(9) \\
19(8)\end{array}$ \\
\hline
\end{tabular}

a Averages of duplicate determinations.

$\mathrm{b} J=$ Cane juice; $\mathrm{C}=$ supplemented cane juice; $\mathrm{O}=$ Oura synthetic medium. The seed yeasts were produced in strongly aerated medium in c Erlenmeyer flasks.

Table 3-Comparative study of short chain fatty acid production by yeasts isolated from rum manufacture

\begin{tabular}{|c|c|c|c|c|c|c|c|c|c|}
\hline \multirow[b]{2}{*}{ Strains } & \multirow[b]{2}{*}{ Mediab } & \multicolumn{8}{|c|}{ Short chain fatty acids (mg/l) } \\
\hline & & Ace tic & Propionic & Acrylic & Isobutyric & Butyric & Isovaleric & Caproic & Totai \\
\hline S. cerevisiae Lb & $\begin{array}{l}\mathrm{J} \\
\mathrm{C} \\
\mathrm{O}\end{array}$ & $\begin{array}{r}96(7)^{c} \\
314(1) \\
390(5)\end{array}$ & $\begin{array}{l}2(15) \\
6(5) \\
1(12)\end{array}$ & $\begin{array}{l}0 \\
0 \\
0\end{array}$ & $\begin{array}{l}3(4) \\
3(8) \\
1(12)\end{array}$ & $\begin{array}{l}0 \\
0.3(9) \\
0.2(8)\end{array}$ & $\begin{array}{l}1(0) \\
1(12) \\
1(16)\end{array}$ & $\begin{array}{l}0.5(12) \\
0.5(10) \\
0.8(9)\end{array}$ & $\begin{array}{l}103(8) \\
325(2) \\
394(6)\end{array}$ \\
\hline S. cerevisiaa 493 & $\begin{array}{l}J \\
c \\
O\end{array}$ & $\begin{array}{l}253(1) \\
201(16) \\
565(8)\end{array}$ & $\begin{array}{l}2(12) \\
4(6) \\
0\end{array}$ & $\begin{array}{l}0 \\
0 \\
0\end{array}$ & $\begin{array}{l}3(0) \\
3(12) \\
2(0)\end{array}$ & $\begin{array}{l}0.2(7) \\
0.4(3) \\
0.3(0)\end{array}$ & $\begin{array}{l}2(8) \\
1(6) \\
1(6)\end{array}$ & $\begin{array}{l}0.8(12) \\
0.4(8) \\
0.8(10)\end{array}$ & $\begin{array}{l}261(2) \\
210(10) \\
570(8)\end{array}$ \\
\hline S. cerevisiae 390 & $\begin{array}{l}J \\
C \\
0\end{array}$ & $\begin{array}{l}148(4) \\
119(9) \\
605(12)\end{array}$ & $\begin{array}{l}2(12) \\
3(8) \\
0\end{array}$ & $\begin{array}{l}0 \\
0 \\
0\end{array}$ & $\begin{array}{l}3(0) \\
3(0) \\
2(0)\end{array}$ & $\begin{array}{l}0.3(16) \\
0.4(10) \\
0.3(8)\end{array}$ & $\begin{array}{l}1(8) \\
1(0) \\
1(8)\end{array}$ & $\begin{array}{l}0.9(15) \\
0.5(7) \\
0.9(10)\end{array}$ & $\begin{array}{l}156(6) \\
127(8) \\
610(12)\end{array}$ \\
\hline S. chevalieri 377 & $\begin{array}{l}\mathrm{J} \\
\mathrm{C} \\
\mathrm{O}\end{array}$ & $\begin{array}{l}550(11) \\
580(15) \\
625(12)\end{array}$ & $\begin{array}{l}4(74) \\
8(6) \\
0\end{array}$ & $\begin{array}{l}0 \\
0 \\
0\end{array}$ & $\begin{array}{l}4(5) \\
4(8) \\
2(12)\end{array}$ & $\begin{array}{l}0.2(5) \\
0.3(10) \\
0.4(12)\end{array}$ & $\begin{array}{l}2(6) \\
1(12) \\
1(7)\end{array}$ & $\begin{array}{l}0.7(4) \\
0.4(12) \\
0.7(12)\end{array}$ & $\begin{array}{l}561(12) \\
594(12) \\
629(12)\end{array}$ \\
\hline S. chevalieri 467 & $\begin{array}{l}J \\
C \\
O\end{array}$ & $\begin{array}{l}238(7) \\
252(9) \\
680(5)\end{array}$ & $\begin{array}{l}3(13) \\
3(8) \\
0\end{array}$ & $\begin{array}{l}0 \\
0 \\
0\end{array}$ & $\begin{array}{l}3(0) \\
3(0) \\
2(0)\end{array}$ & $\begin{array}{l}0.2(0) \\
0.3(10) \\
0.6(9)\end{array}$ & $\begin{array}{l}2(14) \\
1(8) \\
1(0)\end{array}$ & $\begin{array}{l}0.8(7) \\
0.5(10) \\
0.9(14)\end{array}$ & $\begin{array}{l}247(8) \\
265(10) \\
685(6)\end{array}$ \\
\hline Sch. pombe G & $\begin{array}{l}J \\
C \\
O\end{array}$ & $\begin{array}{r}114(3) \\
145(6) \\
67(6)\end{array}$ & $\begin{array}{l}1(12) \\
5(14) \\
0\end{array}$ & $\begin{array}{l}1(12) \\
2(14) \\
0\end{array}$ & $\begin{array}{l}0.5(7) \\
0.4(0) \\
0.2(12)\end{array}$ & $\begin{array}{l}0.1(5) \\
0 \\
0.1(0)\end{array}$ & $\begin{array}{l}0 \\
0 \\
0.2(6)\end{array}$ & $\begin{array}{l}0.4(14) \\
0.6(12) \\
0.4(7)\end{array}$ & $\begin{array}{r}117(4) \\
133(7) \\
68(6)\end{array}$ \\
\hline Sch. pombe 850 & $\begin{array}{l}J \\
C \\
0\end{array}$ & $\begin{array}{l}184(3) \\
204(6) \\
180(8)\end{array}$ & $\begin{array}{l}1(16) \\
2(9) \\
0\end{array}$ & $\begin{array}{l}1(10) \\
3(9) \\
0\end{array}$ & $\begin{array}{l}0.5(4) \\
0.3(8) \\
0.3(4)\end{array}$ & $\begin{array}{l}0.1(0) \\
0.1(7) \\
0.1(0)\end{array}$ & $\begin{array}{l}0.2(8) \\
0.1(12) \\
0.9(18)\end{array}$ & $\begin{array}{l}0.5(10) \\
0.6(7) \\
0.7(11)\end{array}$ & $\begin{array}{l}188(3) \\
212(7) \\
182(9)\end{array}$ \\
\hline
\end{tabular}

a Averages of duplicate determinations

$\mathrm{J}=$ Cane juice; $\mathrm{C}=$ supplemented cane juice; $\mathrm{O}=$ Oura synthetic medium. The seed yeasts were produced in strongly aerated medium in Erlenmeyer flasks.

c $\%$ Relative standard deviation ( $\left.=\frac{\text { standard deviation }}{\text { mean }} \times 1.00\right)$

obtained in rum manufacture (about $6 \mathrm{~g} / \mathrm{L}$ ), which is considerable for an alcoholic fermentation. It should be noted that the cane media were buffered. At the end of the fermentation period, the $\mathrm{pH}$ was close to 3.5 with the Saccharomyces strains and close to 4.0 for Schizosaccharomyces strains. The $\mathrm{pH}$ at the end of the fermentation period was close to 2.8 for the unbuffered Oura medium. Fermenta- tion proceeded faster on the supplemented cane juice than on the pure cane juice. The end of the fermentation period was determined by assaying for residual reducing sugars with the Clinitest (Miles Laboratories Inc.). The longest fermentation periods were observed for the Oura medium. Saccharomyces strains had much shorter fermentation periods than Schizosaccharomyces strains. 
Table 4-Effect of the organic acids on the production of biomass, the fermentation period and the production of higher alcohols

\begin{tabular}{|c|c|c|c|c|c|c|c|c|}
\hline \multirow[b]{2}{*}{ Strains } & \multirow[b]{2}{*}{ Mediab } & \multirow{2}{*}{$\begin{array}{l}\text { Yeast dry } \\
\text { matter } \\
(\mathrm{g} / \mathrm{L})\end{array}$} & \multirow{2}{*}{$\begin{array}{c}\text { Period } \\
\text { (hr) }\end{array}$} & \multicolumn{5}{|c|}{ Higher alcohols (mg/i) } \\
\hline & & & & Propanol & Isobutanol & Butanol & Isopentanols & Total \\
\hline S. cerevisiae Lb & $\begin{array}{l}\text { O } \\
A \\
C \\
M \\
S\end{array}$ & $\begin{array}{l}2.7(1.0)^{c} \\
5.2(2.0) \\
3.5(1.5) \\
3.4(2.5) \\
5.1(2.0)\end{array}$ & $\begin{array}{l}92(2.0) \\
78(3.0) \\
74(2.5) \\
92(3.0) \\
72(2.0)\end{array}$ & $\begin{array}{l}36(8) \\
35(10) \\
32(7) \\
31(12) \\
29(13)\end{array}$ & $\begin{array}{c}6(18) \\
5(14) \\
17(8) \\
21(25) \\
10(9)\end{array}$ & $\begin{array}{l}1(0) \\
2(10) \\
1(12) \\
1(6) \\
1(0)\end{array}$ & $\begin{array}{l}34(7) \\
19(16) \\
37(12) \\
37(10) \\
24(17)\end{array}$ & $\begin{array}{l}77(9) \\
61(12) \\
87(10) \\
99(14) \\
64(14)\end{array}$ \\
\hline S. cerevisiae 493 & $\begin{array}{l}O \\
A \\
C \\
M \\
S\end{array}$ & $\begin{array}{l}2.8(3.0) \\
4.9(1.0) \\
3.5(1.0) \\
3.0(0.5) \\
6.0(2.0)\end{array}$ & $\begin{array}{r}100(1.0) \\
65(2.0) \\
75(1.5) \\
80(1.5) \\
58(0.0)\end{array}$ & $\begin{array}{l}21(13) \\
30(16) \\
23(11) \\
28(17) \\
34(19)\end{array}$ & $\begin{array}{l}12(13) \\
16(9) \\
16(8) \\
18(16) \\
16(13)\end{array}$ & $\begin{array}{l}2(6) \\
2(0) \\
1(0) \\
1(13) \\
2(12)\end{array}$ & $\begin{array}{l}34(10) \\
44(10) \\
40(14) \\
37(6) \\
47(13)\end{array}$ & $\begin{array}{l}69(11) \\
92(12) \\
80(12) \\
84(9) \\
99(14)\end{array}$ \\
\hline S. chevalieri 377 & $\begin{array}{l}O \\
A \\
C \\
M \\
S\end{array}$ & $\begin{array}{l}3.8(1.5) \\
4.5(2.0) \\
3.1(1.0) \\
2.9(1.5) \\
5.1(2.0)\end{array}$ & $\begin{array}{l}72(2.0) \\
80(1.5) \\
84(2.0) \\
84(3.0) \\
76(2.0)\end{array}$ & $\begin{array}{l}25(7) \\
24(12) \\
24(13) \\
24(5) \\
26(9)\end{array}$ & $\begin{array}{l}16(20) \\
20(11) \\
27(16) \\
22(10) \\
21(9)\end{array}$ & $\begin{array}{l}2(6) \\
1(6) \\
1(0) \\
1(12) \\
2(16)\end{array}$ & $\begin{array}{l}42(14) \\
42(9) \\
47(10) \\
47(9) \\
41(6)\end{array}$ & $\begin{array}{l}85(13) \\
89(10) \\
99(12) \\
94(9) \\
90(8)\end{array}$ \\
\hline Sch. pombe G & $\begin{array}{l}O \\
A \\
C \\
M \\
S\end{array}$ & $\begin{array}{l}2.9(1.0) \\
3.5(0.5) \\
3.3(0.0) \\
3.2(1.0) \\
4.0(2.0)\end{array}$ & $\begin{array}{l}140(3.0) \\
125(5.0) \\
140(4.0) \\
145(4.0) \\
140(3.0)\end{array}$ & $\begin{array}{c}8(13) \\
9(7) \\
8(9) \\
8(6) \\
11(9)\end{array}$ & $\begin{array}{l}0 \\
0 \\
0 \\
0 \\
0\end{array}$ & $\begin{array}{l}0 \\
1(20) \\
0 \\
0 \\
0\end{array}$ & $\begin{array}{l}0 \\
2(11) \\
0 \\
0 \\
0\end{array}$ & $\begin{array}{c}8(13) \\
12(12) \\
8(9) \\
8(6) \\
11(9)\end{array}$ \\
\hline
\end{tabular}

a Averages of duplicate determinations

${ }^{b} \mathrm{O}=$ Reference Oura medium; $\mathrm{A}=0+1$ cis-aconitic acid $(1.3 \mathrm{~g} / \mathrm{L}) ; \mathrm{C}=0+$ citric acid $(0.3 \mathrm{~g} / \mathrm{L}) ; \mathrm{M}=0+\mathrm{malic}$ acid $(0.13 \mathrm{~g} / \mathrm{L}) ; \mathrm{S}=0+$ all threc

$c \%$ Relative standard deviation ( $\left.=\frac{\text { standard deviation }}{\text { mean }} \times 100\right)$

Production of higher alcohols and of short chain fatty acids

The production of higher alcohols by Saccharomyces strains appeared to be much greater than that by Schizosaccharomyces strains (Table 2). The latter produced little isopentanols but more propanol among higher alcohols when compared to the Saccharomyces strains. Production of butanol remained slight for all strains and all media, which agrees with the results of Parfait and Jouret (1975). The production of higher alcohols was not qualitatively affected by the use of the synthetic medium. Supplementation of the cane juice medium increased the production of propanol, isobutanol and isopentanols, and consequently, increased the total amount of higher alcohol production.

The production of short chain fatty acids (Table 3 ) was not stimulated by the supplementation of cane juice, except for the commercial baker's yeast. On the Oura medium, the Saccharomyces strains produced more fatty acids than on the cane juice media. The fatty acids production by the Schizosaccharomyces strains was weaker than that by the Saccharomyces strains and was also less on the Oura medium than on the cane juice media. Acetic acid represented more than $90 \%$ of the short chain fatty acids; these acids constituted the greatest part of the volatile acidity in rums from the French West-Indies (Fahrasmane et al., 1983). Only small amounts of other short chain fatty acids were produced. Except for the commercial Saccharomyces cerevisiae $\mathbf{L b}$ strain, which produced propionic acid even when grown on the Oura medium, the other strains only produced this acid when grown on cane juice. The average amount of propionic acid produced was observed to increase when the supplemented cane juice medium was used. Acrylic acid was only produced by the Schizosaccharomyces strains. Valeric and isocaproic acids were not produced.

\section{Influence of the organic acids}

Cane juice and molasses are particularly rich in cisaconitic acid and citric acid, sometimes they also contain malic acid (Matsui, 1976; Meade, 1977).
In order to determine the influence of organic acids on the production of higher alcohols and short chain fatty acids, we performed fermentation tests with the Oura medium under standard conditions, with each acid and with a mixture of the acids. The concentration of each acid alone and in the mixture was the same: cis-aconitic acid $1.3 \mathrm{~g} / \mathrm{L}$, citric acid $0.3 \mathrm{~g} / \mathrm{L}$, L-malic acid $0.13 \mathrm{~g} / \mathrm{L}$. Except for $S$. chevalieri in the presence of citric acid, there was an increase (14 to $93 \%$ ) of the biomass produced by the strains during the fermentation process in the presence of citric acid and cis-aconitic acid as compared to the Oura reference medium under standard culture conditions. The mixture of citric acid, cis-aconitic acid and malic acid supplementing the Oura medium reduced the fermentation period of the baker's yeast strain 493 (Table 4).

Taking into account the standard deviation for duplicate determinations, the production of higher alcohols was not significantly modified by organic acids, except with $S a c$ charomyces cerevisiae $\mathbf{L b}$ for the production of isobutanol in presence of citric and malic acids alone. The three acids added, and especially their mixture, decreased the amount of acetic acid produced by Saccharomyces strains (Table 5). These organic acids had little effect on the metabolism of Schizosaccharomyces, which produced only small amounts of fatty acids. A relatively greater proportion of volatile acids other than acetic was produced in the presence of citric acid by the 493 and $G$ strains, and to a lesser degree by the $\mathrm{Lb}$ strain. No change in fatty acid production was observed for strain $S$. chevalieri 377 in the presence of citric acid.

\section{Study of the effect of branched amino acids}

For this study, we replaced half of the nitrogen source in the Oura medium $(250 \mathrm{~g} / \mathrm{L})$ with amino nitrogen as valine, leucine, isoleucine or a mixture of these three amino acids. The results showed that the decomposition and utilization of amino-nitrogen result in the accumulation of corresponding short chain fatty acids and alcohols in agreement with Suomalainen and Keranen (1967), and fit the catabolic schemes proposed by Rodwell (1969). 
Table 5-Effect the organic acids on the production of short chain fatty acids ${ }^{a}$

\begin{tabular}{|c|c|c|c|c|c|c|c|c|c|c|c|c|}
\hline \multirow[b]{2}{*}{ Strains } & \multirow[b]{2}{*}{ Media $^{b}$} & \multicolumn{11}{|c|}{ Short chain fatty acids $(\mathrm{mg} / \mathrm{l})$} \\
\hline & & Acetic & Propionic & Acrylic & Isoblityric & Butyric & Isovaleric & Valeric & Isocaproic & Caproic & Total & $\begin{array}{l}\text { Acetic } \\
\text { acid } \\
\% \text { total }\end{array}$ \\
\hline S. cerevisiae Lb & $\begin{array}{l}O \\
A \\
C \\
M \\
S\end{array}$ & $\begin{array}{l}770(6)^{c} \\
530(7) \\
340(4) \\
480(7) \\
320(9)\end{array}$ & $\begin{array}{l}0 \\
0 \\
2(12) \\
0 \\
0\end{array}$ & $\begin{array}{l}0 \\
0 \\
1(12) \\
0 \\
0\end{array}$ & $\begin{array}{l}1(2) \\
1(6) \\
2(6) \\
1(12) \\
2(13)\end{array}$ & $\begin{array}{l}0.3(6) \\
0.1(12) \\
2(15) \\
0.1(12) \\
0\end{array}$ & $\begin{array}{l}1(8) \\
0.4(14) \\
2(9) \\
0.4(6) \\
0.6(6)\end{array}$ & $\begin{array}{l}0 \\
0 \\
2(11) \\
0 \\
0\end{array}$ & $\begin{array}{l}0 \\
0 \\
0.5(10) \\
0 \\
0\end{array}$ & $\begin{array}{l}0.5(8) \\
0.4(10) \\
2(12) \\
0.3(12) \\
0.6(16)\end{array}$ & $\begin{array}{l}773(6) \\
532(8) \\
354(5) \\
483(8) \\
324(10)\end{array}$ & $\begin{array}{l}99 \\
99 \\
96 \\
99 \\
99\end{array}$ \\
\hline S. cerevisiae 493 & $\begin{array}{l}0 \\
A \\
C \\
M \\
S\end{array}$ & $\begin{array}{l}616(5) \\
310(13) \\
390(7) \\
590(7) \\
230(12)\end{array}$ & $\begin{array}{l}0 \\
0 \\
9(12) \\
0 \\
0\end{array}$ & $\begin{array}{l}0 \\
0 \\
8(10) \\
0 \\
0\end{array}$ & $\begin{array}{l}2(8) \\
2(10) \\
8(3) \\
3(7) \\
2(8)\end{array}$ & $\begin{array}{l}0.2(6) \\
0.3(8) \\
6(12) \\
0.3(8) \\
0.3(8)\end{array}$ & $\begin{array}{l}1(12) \\
0.8(16) \\
6(9) \\
0.8(12) \\
1(14)\end{array}$ & $\begin{array}{l}0 \\
0 \\
7(8) \\
0 \\
0\end{array}$ & $\begin{array}{l}0 \\
0 \\
5(7) \\
0 \\
0\end{array}$ & $\begin{array}{l}0.9(14) \\
0.7(6) \\
7(8) \\
0.7(10) \\
0.3(12)\end{array}$ & $\begin{array}{l}640(5) \\
314(13) \\
447(7) \\
595(7) \\
335(12)\end{array}$ & $\begin{array}{l}99 \\
98 \\
87 \\
99 \\
98\end{array}$ \\
\hline S. chevalieri 377 & $\begin{array}{l}O \\
A \\
C \\
M \\
S\end{array}$ & $\begin{array}{l}760(7) \\
320(12) \\
540(3) \\
440(6) \\
570(9)\end{array}$ & $\begin{array}{l}0 \\
1(16) \\
0 \\
0 \\
0\end{array}$ & $\begin{array}{l}0 \\
0 \\
0 \\
0 \\
0\end{array}$ & $\begin{array}{l}2(7) \\
2(6) \\
3(11) \\
3(8) \\
2(12)\end{array}$ & $\begin{array}{l}0.3(9) \\
0.3(11) \\
0.2(15) \\
0.2(12) \\
0.2(12)\end{array}$ & $\begin{array}{l}1(7) \\
0.9(12) \\
1(12) \\
0.9(10) \\
1(6)\end{array}$ & $\begin{array}{l}0 \\
0 \\
0.1 \\
0 \\
0\end{array}$ & $\begin{array}{l}0 \\
0 \\
0 \\
0 \\
0\end{array}$ & $\begin{array}{l}0.7(14) \\
0.6(10) \\
1(8) \\
0.8(12) \\
0.7(16)\end{array}$ & $\begin{array}{l}764(8) \\
525(12) \\
546(4) \\
445(6) \\
573(9)\end{array}$ & $\begin{array}{l}99 \\
99 \\
99 \\
99 \\
99\end{array}$ \\
\hline Sch. pombe G & $\begin{array}{l}O \\
A \\
C \\
M \\
S\end{array}$ & $\begin{array}{c}91(6) \\
110(7) \\
83(7) \\
120(11) \\
111(9)\end{array}$ & $\begin{array}{l}0 \\
0 \\
6(8) \\
0 \\
0\end{array}$ & $\begin{array}{l}0 \\
0 \\
6(7) \\
0 \\
0\end{array}$ & $\begin{array}{l}0.1(10) \\
0.1(16) \\
4(12) \\
0 \\
0.1(12)\end{array}$ & $\begin{array}{l}0 \\
0 \\
3(11) \\
0 \\
0\end{array}$ & $\begin{array}{l}0 \\
0 \\
4(9) \\
0.2(8) \\
0\end{array}$ & $\begin{array}{l}0 \\
0 \\
5(13) \\
0 \\
0\end{array}$ & $\begin{array}{l}0 \\
0 \\
4(15) \\
0 \\
0\end{array}$ & $\begin{array}{l}0.8(9) \\
0.5(14) \\
6(7) \\
0.3(8) \\
0.5(12)\end{array}$ & $\begin{array}{l}92(6) \\
111(7) \\
123(8) \\
121(11) \\
112(9)\end{array}$ & $\begin{array}{l}99 \\
99 \\
67 \\
99 \\
99\end{array}$ \\
\hline
\end{tabular}

a Means of duplicate determinations

${ }^{b} O=$ Référence Oura medium; $A=0+1$ cis-aconitic acia $(1.3 \mathrm{~g} / L) ; C=0+$ citric acid $(0.3 \mathrm{~g} / \mathrm{L}) ; \mathrm{M}=0+\mathrm{malic}$ acid $(0.13 \mathrm{~g} / \mathrm{L}) ; \mathrm{S}: 0+\mathrm{all}$ three acids. $\left.\frac{\text { standard deviation }}{\operatorname{mean}} \times 100\right)$

\section{DISCUSSION}

THE SCHIZOSACCHAROMYCES STRAINS produced less higher alcohols and short chain fatty acids than the Saccharomyces strains. Large differences were observed in the production of acetic acid between strains of the same species. The type of medium seemed to influence only secondarily the production of higher alcohols. On the other hand the media appeared to have a role in the production of short chain fatty acids both quantitatively and qualitatively (Table 3 and 5). Suomalainen (1975) reported there was more acetic acid in rum than in whisky or brandy. Fahrasmane et al. (1983) observed the presence of acrylic acid in some types of rum.

The reduction of the fermentation period observed in the presence of citric acid, aconitic acid and their mixture was probably due to the buffering effect of these compounds. The resulting final $\mathrm{pH}$ was from 0.3 to 0.4 higher than the standard Oura medium fermentation.

The branched short chain fatty acids are mainly produced from the oxydative decarboxylation of corresponding $\alpha$-keto-acids which are metabolic by-products of valine, leucine, and isoleucine metabolism (Suomalainen and Ronkanen, 1963). They are probably the starting elements for the synthesis of longer branched acids such as isocaproic acid. The presence of the organic acids which promote better cell growth could thus indirectly have an influence on the formation of fatty acids. Taylor and Kirsop (1977) showed that the medium long linear acids $\left(C_{6}\right.$ to $\left.C_{10}\right)$ were produced by biosynthesis rather than by degradation during the brewing fermentation process. The responsible metabolism for the formation of short chain fatty acids by yeast has not been studied extensively. It is reasonable to suppose that the short chain fatty acids are formed in a manner similar to the medium chain fatty acids during the biosynthesis of long chain fatty acids. It is known that the deacylase responsible for the liberation of fatty acids during their biosynthesis liberates mainly palmitic acid $\left(\mathrm{C}_{16}\right)$ and longer fatty acids. In some plants (coconut, palm tree) and in the mammary gland, this enzyme liberates shorter fatty acids. A similar mechanism may be present in yeast. Citric acid plays an important role in the synthesis of fatty acids by its transport through mitochondrial membrane and by allosteric activation of acetyl-CoA-carboxylase. Isocitrate and fluoroacetate also have the same activation effect. Notwithstanding the mechanism, citric acid appeared clearly to modify both quantitatively and qualitatively the production of short chain fatty acids.

It is probable that the mechanism is complex since the mixture of citric, aconitic, and malic acids did not show the effects observed with citric acid alone. Stadtman (1956) showed that bacteria could transform propionic acid into acrylic acid. The formation of acrylic acid in the presence of citric acid by Saccharomyces cerevisiae and Schizosaccharomyces pombe must be an indirect consequence of the formation of propionic acid. It is also noteworthy that citric acid induced the production of three other fatty acids normally absent from the fermentation medium: propionic, valeric, and isocaproic acids.

The action of citric acid is complex and its content in cane juice and molasses may be an element for the characteristics of rum.

The composition of the fermented product, and in this case the higher alcohols and fatty acids contents, depends on the interaction of the constituents of the raw materials (Montreau and Dufrenot, 1974) and the general metabolism of the dominant yeast strains. Acrylic acid appeared to be produced by yeast through an unknown mechanism influenced by citric acid which plays an important role in the formation of short chain fatty acids. The presence of propionic and acrylic acids is particular to rum (Suomalainen, 1975; Fahrasmáne et al., 1983).

\section{SUMMARY}

THE SACCHAROMYCES STRAINS fermented the media tested faster than the Schizosaccharomyces strains and produced more higher alcohols and more short chain fatty acids than the Schizosaccharomyces strains.

The presence of organic acids, and especially of citric 